\title{
Cardiac Motion Estimation in Multislice Computed Tomography Imaging Using a 4D Multiscale Surface-Volume Matching Process
}

\author{
A Simon ${ }^{1}$, M Garreau ${ }^{1}$, D Boulmier ${ }^{2}$, C Toumoulin ${ }^{1}$, H Le Breton ${ }^{1,2}$ \\ ${ }^{1}$ LTSI, INSERM U642, Université de Rennes 1, Campus de Beaulieu, 35042 Rennes, France \\ ${ }^{2}$ Centre Cardio-Pneumologique, CHU Pontchaillou, 35033 Rennes, France
}

\begin{abstract}
A new generation of Multislice Computed Tomography (MSCT) scanners, which allow a complete heart coverage, is becoming widely available. With retrospective data reconstruction, it offers new perspectives for cardiac kinetics evaluation in non invasive imaging with volume sequences of high spatial and temporal resolutions. A new method is proposed for cardiac motion extraction in MSCT. It is based on a 3D surface-volume matching process associated to a hierarchical description of shapes. Needing the segmentation of only one volume, it provides, on a whole dynamic sequence, $3 D$ velocity fields associated to the left ventricle inner surface and an estimation of the localization of this surface. The estimated motion can be used for global and local functional parameters quantification. Results obtained on simulated and real data show the good behavior of this method.
\end{abstract}

\section{Introduction}

Cardiovascular diseases represent the major cause of mortality in industrialized countries. Different cardiac functional parameters are used to guide diagnosis, treatment and follow-up of these diseases. The most used indicators provide information on the heart global function, but the detection and the treatment of some pathologies would need the precise quantification of cardiac motion and deformation.

Because the heart is a nonrigid object that deforms in 3D space, only 3D representation based methods can provide accurate motion descriptors. Image processing methods, applied on 3D cardiac dynamic sequences, can be used to quantify heart motion.

Assessment of heart motion with minimally invasive modalities has been studied from four-dimensional (3D+T) data sets with magnetic resonance (MR) imaging [1] (and especially MR tagging [2, 3]), transthoracic ultra-sound images [4, 5] and the Dynamic Spatial Reconstructor (DSR) $[6,7,8,9]$. See [10] for a more exhaustive review of cardiac image functional analysis methods.

The recent significant advances of spiral computed tomography, with the introduction of ultra-fast rotating gantries, multi-rows detectors and retrospective ECGgated reconstructions, provide higher contrast and spatiotemporal resolutions and allow a huge progress toward the imaging of moving organs. Some first reference studies have been conducted in MSCT for the automatic detection of coronary diseases $[11,12]$ and some first clinical studies have been realized for cardiac functional analysis [13], but very few works have been developed for the quantitative 3D cardiac motion automatic estimation [14].

Based on these 4D CT images, a new method is proposed to extract non-rigid motion of $3 \mathrm{D}$ structures and is applied to the left ventricle. This estimation method extracts the motion between a 3D segmented surface which has been reconstructed at one time and the volume image corresponding to the next time. A surface estimation process, using the extracted motion, provides the localization of the surface corresponding to the second time. This way to proceed enables to apply the segmentation tool only to the first moment of the sequence, providing a method which avoids segmentation temporal coherence problems.

\section{Method}

\subsection{Global process}

The issue is to compute the motion, and to estimate the localization, of the endocardial surface represented in a dynamic sequence of 3D volumes. The global process involves three main steps:

(a) a preprocessing step, including a segmentation, a surface reconstruction and a surface regularization processes; (b) a hierarchical motion estimation step, applied between the surface corresponding to the considered time $t_{1}$ and the volume corresponding to the following time $t_{2}$;

(c) a surface estimation step resulting in the surface corresponding to time $t_{2}$.

Step (a) is applied only to the first considered volume. Steps (b) and (c) are iteratively applied to the whole se- 
quence in order to estimate both the cardiac motion and the endocardial surface during a whole cardiac cycle.

In order to obtain the mesh corresponding to the first considered time of the sequence, step (a) is decomposed in this way: a segmentation tool, based on a $3 \mathrm{D}$ region growing process bounded by a gradient information, is applied [15]. The segmented surface is then reconstructed using the Marching Cubes algorithm. Finally, in order to prepare the matching process, the resulting surface mesh is regularized in such a manner that each node coordinates correspond to one volume voxel coordinates.

In order to obtain the surface corresponding to time $t_{2}$, the surface estimation step (c) relies on the deformation of the surface corresponding to time $t_{1}$ with the motion estimated between times $t_{1}$ and $t_{2}$. A regularization step is then performed in order to fill mesh holes and to suppress redundant nodes.

\subsection{Hierarchical motion estimation}

The 3D motion field estimation (step (b)) is performed by a matching process applied between the surface representing the endocardium at time $t_{1}$ and the original volume corresponding to the next time $t_{2}$. A hierarchical process is used to gain both in terms of result quality and of computational efficiency.

The matching process will firstly be described at one resolution, then the multiresolution scheme will be detailed.

\subsubsection{Matching process}

The 3D motion field to compute between two successive instants is considered as a realization $f=$ $\left\{f_{i} / i=1, \ldots, N_{S}\right\}$ of a 3D random field $F\left(N_{S}\right.$ being the number of considered sites in the field). The set of sites $S$ of the field $F$ is given by all the 3D mesh nodes at time $t_{1}$. The labels assigned to these sites, expressed by the $f_{i}$ estimations, are given by the voxels found (at time $t_{2}$ ) in best correspondence with the $3 \mathrm{D}$ nodes.

According to Bayes' theory, the posterior probability of the realization $f$ according to the observation $d$ (the mesh nodes at time $t_{1}$ and the voxels at time $t_{2}$ ) is given by:

$$
\mathrm{P}(f \mid d)=\frac{\mathrm{P}(f) \mathrm{P}(d \mid f)}{\mathrm{P}(d)},
$$

with $\mathrm{P}(f)$ the prior probability, $\mathrm{P}(d \mid f)$ the conditional probability of the observation process $d$ and $\mathrm{P}(d)$ the observation probability which is considered independent of $f$. According to the maximum a posterior (MAP) estimator, the most probable realization $f$ is provided by the maximisation of the posterior probability $\mathrm{P}(f \mid d)$, with $\mathrm{P}(f \mid d) \propto \mathrm{P}(f) \mathrm{P}(d \mid f)$.

The mechanical properties of the heart induce a spatiotemporal regularity of the motion field. To capture this characteristic, the random field $F$ is modeled by a Markov Random Field (MRF) in relation to a neighbourhood system $\mu$ : the local neighbourhood $\mu_{i}$ of each node $i$ is given by all nodes $j$ which have a common edge with $i$.

According to the Hammersley-Clifford theorem and assuming that $\mathrm{P}(f)>0$, the random field $F$ is a Gibbs Random Field in relation to the neighbourhood system $\mu$. Therefore, the probability distribution function is given by:

$$
\mathrm{P}(f)=\frac{1}{Z} \exp \left\{-U_{r}(f)\right\}=\frac{1}{Z} \exp \left\{-\sum_{c \in C} V_{c}\left(f_{c}\right)\right\}
$$

with $U_{r}(f)$ the field internal energy function, defining the interactions between the field sites and having a regularization effect, and $Z$ a normalization constant. The interaction potentials $V_{c}(c \in C)$ are defined for each clique $c$ belonging to the cliques set $C$. $C$ is defined as all pairs of neighbouring nodes and $f_{c}=\left\{f_{i}, i \in c\right\} . V_{c}$ is given by:

$$
V_{c}\left(f_{c}\right)=\frac{\left\|\overrightarrow{f_{s}}-\overrightarrow{f_{t}}\right\|}{\operatorname{dist}(s, t)} \forall c=\{s, t\} \in C
$$

where $\overrightarrow{f_{s}}\left(\right.$ resp. $\left.\overrightarrow{f_{t}}\right)$ is the motion vector estimated at site $s$ (resp. $t$ ).

The conditional probability of the observation process is provided by the data model:

$$
\mathrm{P}(d \mid f)=\exp \left\{-U_{d}(f, d)\right\}=\exp \left\{-\sum_{i \in S} V_{i}\left(n_{i}, f_{i}\right)\right\} .
$$

$U_{d}(f, d)$ models the estimation error with:

$$
\begin{aligned}
V_{i}\left(n_{i}, f_{i}\right)= & \alpha_{p} \cdot E_{p}\left(n_{i}, f_{i}\right)+\alpha_{t} \cdot E_{t}\left(n_{i}, f_{i}\right) \\
& +\alpha_{c} \cdot E_{c}\left(f_{i}\right),
\end{aligned}
$$

where $E_{p}\left(n_{i}, f_{i}\right)$ is the Euclidean distance between node $n_{i}$ and voxel $f_{i}$, and $E_{c}\left(f_{i}\right)$ corresponds to the belonging of voxel $f_{i}$ to a contour (estimated by a Canny filter). $E_{t}\left(i, f_{i}\right)$ expresses a topological correspondence between the node $i$ and the voxel $f_{i}$ : the topology is described, for the surface, in terms of coordinates of the neighbouring nodes and compared, in the volume, to the corresponding voxel contour values.

Finally, the total energy to minimize, noted $U(f, d)$, is thus defined by the linear combination of two terms:

$$
U(f, d)=U_{d}(f, d)+\alpha_{1} U_{r}(f) .
$$

\subsubsection{Hierarchical scheme}

The previously described motion extraction process is applied according to a hierarchical scheme which allows to focus the correspondence research area and to reduce computing needs. This multiresolution scheme is considered to preserve local Markov property according to the high spatial resolution provided by MSCT data. 
The surface mesh at time $t_{1}$ and the volume at next time $t_{2}$ are defined with decreasing scales as follows: each data set from upper resolution $R_{i}\left(i=1, \ldots, n_{l}, n_{l}\right.$ being the number of used resolution levels) is restricted in space at a lower level scale $R_{i-1}$ by the application of a mean filtering (or Gaussian filtering for the volume) and of a subsampling process in order to provide a regular mesh corresponding to volume voxel coordinates at the same level. The matching process is first applied at the lowest resolution (with an initialization to a null motion) to guide the motion estimation with the coarsest details. The result of that first estimation is used as an initialization, after an interpolation step, for the correspondences computation at the next finer resolution. This extraction motion process is applied iteratively, with an adaptation of energy weighting coefficients, until the estimation is obtained at the desired resolution.

The global optimization of the correspondences is performed with a stochastic relaxation Metropolis algorithm combined with a simulated annealing process at the first lower resolution level and with an Iterated Conditional Mode (ICM) algorithm at the upper resolution levels.

\section{Results}

\subsection{Tests on simulated data}

Numerical simulations have been used to test the motion extraction process between two successive instants. In order to obtain the simulated data, a segmented endocardial surface, corresponding to the first instant, is deformed using five kinds of motion (translation, twisting, rotation, global expansion/contraction and local deformations) resulting in the mesh corresponding to the second instant. Then, this deformed mesh is inserted into a volume preprocessed by a Canny filter followed by an endocardial suppression step. The hierarchical matching process is finally applied between the surface before deformation and this volume at three increasing scales.

Using this process, the real correspondences are known. It enables to measure the error of matching of the proposed method and to study the evolution of the matching process along iterations and at each stage of the hierarchical process. The impact of the different parameters involved in the computation of the energies or in the optimization process, as well as meaningful information provided by scale refinement, can also be evaluated.

Figure 1 illustrates an example of results obtained at the two lowest resolution levels $\left(R_{1}\right.$ with volume size $64^{3}$ and $R_{2}\left(128^{3}\right)$ ). In color is represented the applied $(\mathrm{a}, \mathrm{b})$ and estimated $(\mathrm{c}, \mathrm{d})$ motion amplitude. In red are represented the displacements directed inside the cavity. Considering the real voxel size $(0.35 \mathrm{~mm})$, with an initial mean matching error of 10.5 voxels at $R_{1}$ level, the process converges

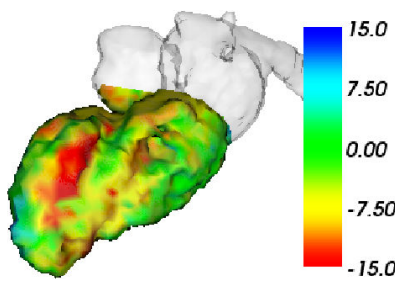

(a)

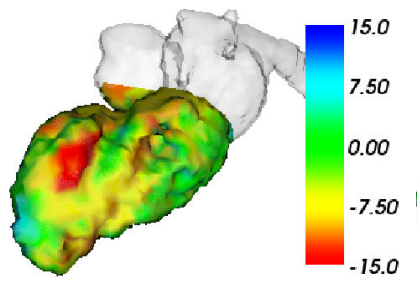

(c)

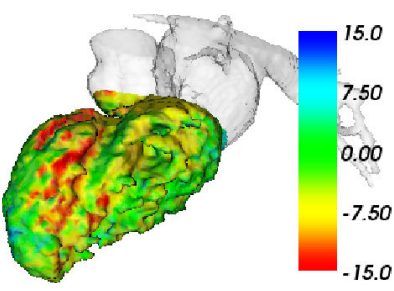

(b)

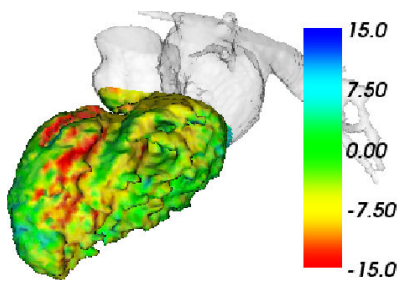

(d)
Figure 1. Simulated $(\mathrm{a}, \mathrm{b})$ and estimated (c,d) motion amplitude with a left oblique anterior view at two resolutions (levels $64^{3}$ (a,c) and $128^{3}(\mathrm{~b}, \mathrm{~d})$ ) (colours: in blue (resp. red): motion directed outside (resp. inside) the cavity), measures in voxels.

to a final mean error of 1.0 voxel at $R_{3}$ level. We have observed that this hierarchical process enables to gain in precision and in error deviation.

\subsection{Results on real data}

The algorithm has been applied on real human heart data with a temporal database acquired by a Siemens SOMATOM Sensation 16 with ten volume images representing a whole cardiac cycle. Each volume contains about 300 slices of $512 \times 512$ pixels, giving a resolution for each voxel of $0.35 \times 0.35 \times 0.5 \mathrm{~mm}$.

The proposed method has been applied to this dynamic sequence considering three resolution levels (from $64^{3}$ to $256^{3}$ ). Figure 2 illustrates results obtained at the two lowest levels at end-diastolic (a,b) and end-systolic (c,d) instants. The contraction movements during systole as well as expansion movements during diastole are identified and coherent with cardiac phases. We can see that the lowest level representation is meaningful and that the displacements obtained at upper levels provide the means to extract local clinical parameters at various scales.

\section{Discussion and conclusions}

A new solution for motion extraction combined with surface estimation has been introduced and applied to the left ventricle in 4D cardiac MSCT imaging. This approach is based on a hierarchical surface-volume matching method formulated with a Markov Random Field and 


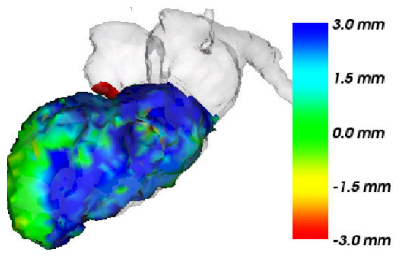

(a)

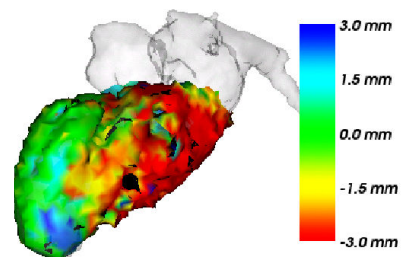

(c)

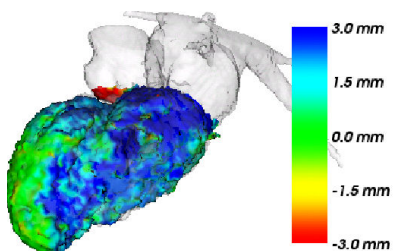

(b)

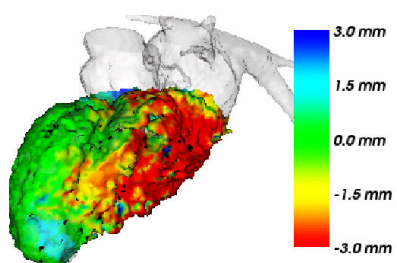

(d)
Figure 2. Estimated motion amplitude at end-diastolic (a,b) and end-systolic (c,d) instants, at two resolutions (levels $64^{3}(\mathrm{a}, \mathrm{c})$ and $\left.128^{3}(\mathrm{~b}, \mathrm{~d})\right)$ (colours: in blue (resp. red): motion directed outside (resp. inside) the cavity).

provides, with one unique process, left cavity surfaces and associated 3D motion vector fields. The algorithm has been tested with simulated and real MSCT dynamic data, highlighting encouraging results and confirming the great potential of MSCT imaging for quantitative clinical measure assessment in cardiac applications. Further works will carry on algorithm optimization and on extensive evaluation.

\section{Acknowledgements}

This work is supported by Brittany region and the French Medical Research and Health National Institute (INSERM). The authors express their thanks to Siemens, Medical Division, France.

\section{References}

[1] Shi P, Sinusas AJ, Constable RT, Ritman E, Duncan JS. Point-tracked quantitative analysis of left ventricular surface motion from 3-D image sequences. IEEE Trans Medical Imaging jan 2000;19:36-50.

[2] Park J, Metaxas D, Young AA, Axel L. Deformable models with parameter functions for cardiac motion analysis from tagged MRI data. IEEE Trans Medical Imaging jun 1996; 15:278-289.

[3] Huang J, Abendschein D, Davila-Roman V, Amini AA. Spatio-temporal tracking of myocardial deformations with a 4D B-spline model from tagged MRI. IEEE Trans Medical Imaging oct 1999;18:957-972.

[4] Maehle J, Bjoernstad K, Aakhus S, Torp HG, Angelsen BA. Three-dimensional echocardiography for quantitative left ventricular wall motion analysis: a method for recon- struction of endocardial surface and evaluation of regional dysfunction. Echocardiography jul 1994;11:397-408.

[5] Papademetris X, Sinusas AJ, Dione DP, Duncan J. Estimation of 3D left ventricular deformation from echocardiography. Medical Image Analysis mar 2001;5:17-28.

[6] Amini AA, Duncan J. Bending and stretching models for LV wall motion analysis from curves and surfaces. Image and Vision Computing July/Aug. 1992;10:418-430.

[7] Benayoun S, Ayache N. Dense non-rigid motion estimation in sequences of medical images using differential constraints. Int Journal of Computer Vision jan 1998;26:25-40.

[8] Gorce JM, Friboulet D, Magnin IE. Estimation of threedimensional cardiac velocity fields: assessment of a differential method and application to three-dimensional CT data. Medical Image Analysis apr 1997;1:245-261.

[9] Eusemann CD, Ritman EL, Robb RA. Parametric visualization methods for the quantitative assessment of myocardial motion. Academic Radiology jan 2003;10:66-76.

[10] Frangi AF, Niessen WJ, Viergever MA. Three-dimensional modeling for functional analysis of cardiac images, a review. IEEE Trans Medical Imaging jan 2001;20:2-25.

[11] Schroeder S, Kopp AF, et al. Accuracy and reliability of quantitative measurements in coronary arteries by multislice computed tomography: experimental and initial clinical results. Clinical Radiology jun 2001;56:466-474.

[12] Larralde A, Boldak C, Garreau M, Toumoulin C, Boulmier D, Rolland Y. Evaluation of a 3D segmentation software for the coronary characterization in multi-slice computed tomography. In Lecture Notes in Computer Science, Functional Imaging and Modeling of the Heart (FIMH'03). Lyon, France, jun 2003; 39-51.

[13] Yamamuro M, Tadamura E, et al. Cardiac functional analysis with multi-detector row $\mathrm{CT}$ and segmental reconstruction algorithm: Comparison with echocardiography, SPECT, and MR imaging. Radiology feb 2005;234:381390.

[14] Garreau M, Simon A, Boulmier D, Guillaume H. Cardiac motion extraction in multislice computed tomography by using a 3D hierarchical surface matching process. In Proc. IEEE Computers in Cardiology (CinC'04). Chicago, USA, sep 2004; 549-552.

[15] Guillaume H, Garreau M. Segmentation de cavités cardiaques en imagerie scanner multi-barettes. In 12ème Forum des Jeunes Chercheurs en Génie Biologique et Médical (2003). Nantes, France, may 2003; 92-93.

Address for correspondence:

Antoine Simon

LTSI, INSERM U642, Université de Rennes 1, Campus de Beaulieu, 35042 Rennes, France antoine.simon@univ-rennes1.fr 